\title{
Reflexes evoked in human thenar muscles during voluntary activity and their conduction pathways
}

\author{
E. F. S T A N L E Y \\ From the School of Biological Sciences, University of East Anglia, Norwich
}

S UMMARY Responses evoked by an electrical stimulus to the median nerve in a small muscle of the human hand during a voluntary contraction have been examined. Two of these responses have been shown to be evoked through reflex pathways. The first, with a mean afferent conduction velocity of $64 \mathrm{~m} / \mathrm{s}$ and estimated central delay of about $0.8 \mathrm{~ms}$, is identified as an $\mathrm{H}$ reflex. The second response, which has an undefined central pathway, has a mean afferent conduction velocity of $43 \mathrm{~m} / \mathrm{s}$ and an estimated central delay of about $17 \mathrm{~ms}$.

Muscle responses evoked by electrical stimuli to nerve trunks in man were first examined by Hoffmann (1918). He showed that a stimulus to the tibial nerve evokes two muscle action potentials (MAPs) in the electromyogram of the calf muscles -an early response caused by direct excitation of motor nerves, termed the $M$ wave, and a late response, termed the $\mathrm{H}$ wave. The $\mathrm{H}$ wave has been shown to be evoked through a monosynaptic reflex pathway (Magladery et al., 1951), and is probably transmitted through the same conduction pathway as the tendon jerk.

$\mathbf{H}$ waves may be recorded in several muscles of the leg and arm but cannot be evoked in the resting intrinsic muscles of the hand except during post-tetanic potentiation (Hagbarth, 1962), in certain disease states (Teasdall et al., 1952), and in infants (Thomas and Lambert, 1960). A small response, termed the $\mathrm{F}$ wave, with a latency similar to the $\mathrm{H}$ wave may be evoked in these muscles but only by a stimulus of suprathreshold intensity for motor nerves. $F$ waves are believed to result from antidromic excitation of the motoneurone cell bodies since they may still be evoked after section of the dorsal roots (Gassel and Wiesendanger, 1965; McLeod and Wray, 1966; Mayer and Feldman, 1967).

Upton et al. (1971) have shown that two late responses of variable amplitude may be recorded in the intrinsic muscles of the hand, after a stimulus to the ulnar or median nerves during a voluntary contraction, with latencies to onset of

Address for reprint requests: Department of Neurology, Johns Hopkins University, School of Medicine, Baltimore, Maryland 21205, USA. Accepted 9 June 1978 approximately $25 \mathrm{~ms}$ and $50 \mathrm{~ms}$. They called theseo responses the V1 and V2 waves. Upton et al. sug-ণ্ণ gested that the first response, the V1 wave, which? has a latency similar to an $F$ wave, was an unmasked $\mathrm{H}$ reflex; in the resting state the motor neurones are excited only subliminally by theo afferent volley initiated by the stimulus, but during $\mathbb{\circ}$ a contraction the excitability of the motor neuroneo pool is raised so that an $\mathrm{H}$ reflex may be evoked. They could not, however, define the origins of the V2 wave but suggested that it was evoked through a polysynaptic pathway.

The origins of the $\mathrm{V} 1$ and $\mathrm{V} 2$ waves are examined further in this study. The results show that both responses are evoked independently of direct excitation of the motor nerves and presumably, therefore, through reflex pathways. The peripheral nerve conduction velocities of the responses are determined and from these the central delays of the reflex arcs are estimated.

\section{Methods}

Seventeen experimental subjects aged between 19 and 30 years were used in the study.

The subject was seated comfortably and his left arm was secured, palm up, to a board by a plate over the fingers. Two $9 \mathrm{~mm}$ silver/silver chloride disc surface recording electrodes were positioned, one over the abductor pollicis brevis (APB) muscle and the other midway along the proximal phallanx of the thumb. Responses recorded by the electrodes were amplified by a differential AC amplifier with a frequency range of $15 \mathrm{~Hz}$ to $5 \mathrm{kHz}$ and displayed on a dual trace independent 
sweep speed oscilloscope (Tetronix type RM565). Traces were photographed on moving film or by a Polaroid camera.

A square wave generator supplied an electric pulse of $1 \mathrm{~ms}$ duration which triggered a $0-100$ volt DC battery powered stimulator through a light/photocell isolator. The stimulating cathode was a $9 \mathrm{~mm}$ silver/silver chloride disc attached to the skin with adhesive tape. The anode was a silver/silver chloride plate, $40 \times 60 \mathrm{~mm}$, and was positioned on the dorsal surface of either the wrist or elbow. Stimuli were of $1.0 \mathrm{~ms}$ duration and were given at a maximum frequency of one per 10 seconds. The subject was earthed by a silver plate attached to the dorsal surface of the hand. Cambridge electrode jelly was used with all electrodes.

The thumb was inserted into a ring which was attached to a Grass (FT 03C) strain gauge. The strain gauge was positioned so that the ring held the thumb flexed, maintaining a small load on the APB muscle at rest. The subject was instructed to contract the thenar muscles, so as to raise the thumb at right angles to the plane of the hand, against the strain gauge ring. When the tension recorded by the strain gauge reached a predetermined value (termed the triggering tension) the stimulus was automatically given and recording of the electromyogram begun. In this way the level of voluntary activity at which the responses were evoked could be controlled. For example in 50 contractions at various rates set at a triggering tension of $0.55 \mathrm{~kg}$ the actual triggering tension ranged by $\pm 8.2 \%$. The subjects were instructed to make the contractions as consistent as possible and to maintain them for about one second. One contraction, and consequently one recording, was made every 10 seconds.

\section{Results}

A stimulus of suprathreshold intensity for motor nerves given to the median nerve at the wrist evoked clear V1 and V2 waves in the surface electromyogram recorded from the APB muscle in most subjects (Fig. 1a).

The V1 and V2 waves could also be evoked in the absence of an $M$ wave at lower stimulus intensities. The relationship between stimulus intensity and the recorded amplitudes of the V1, $\mathrm{V} 2, \mathbf{M}$, and $\mathbf{F}$ waves was examined in seven subjects. At each stimulus intensity 20 traces were recorded during a voluntary contraction with the stimulus triggered automatically by the subject as above, and 10 traces were recorded from the resting muscle with the stimulus triggered by the

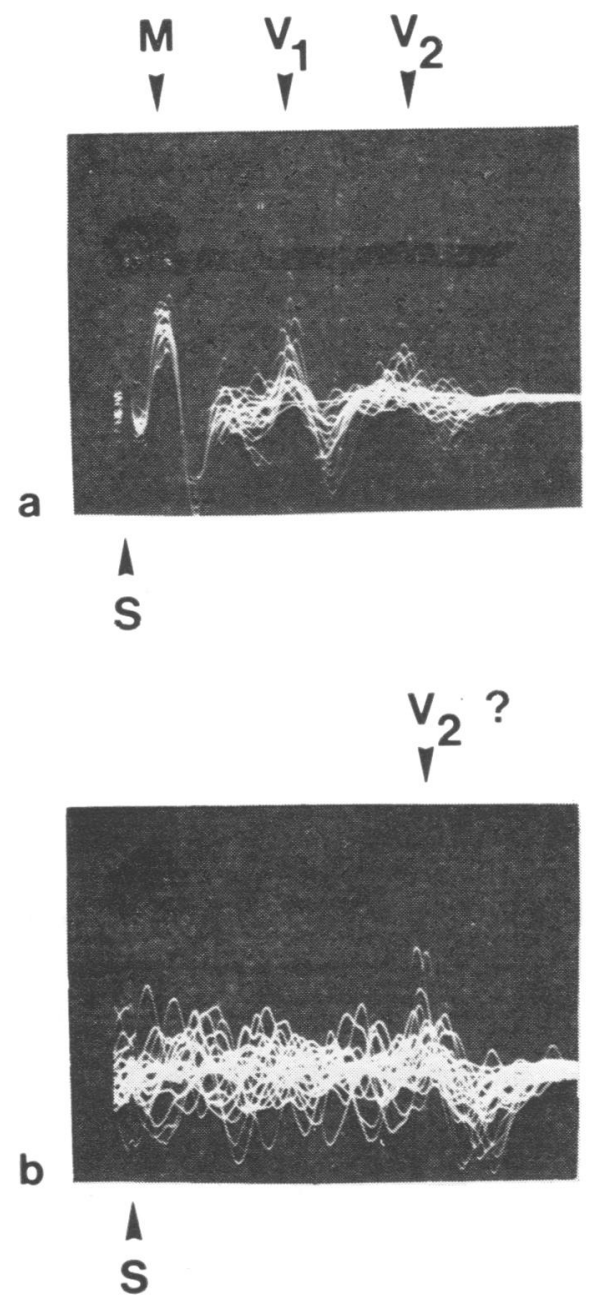

\section{$50 \mathrm{~ms}$}

Fig. 1 Superimposed electromyograms recorded from the APB muscle during a voluntary contraction. (a) Responses recorded after a stimulus of suprathreshold intensity for motor nerves to the median nerve at the wrist. (b) Responses recorded after a stimulus to the radial nerve at the wrist. Note the absence of $M$ and $V 1$ waves and the response (labelled V2? in $b$ ), and the similar latency of this response and the $V 2$ wave in (a). $S=$ stimulus artefact.

experimenter. The mean amplitudes of the V1 and V2 waves were determined from the traces recorded during a contraction, and the mean amplitudes of the $M$ and $F$ waves were obtained from the traces recorded from the resting muscle (Fig. 2).

In order to determine the excitability curves of the V1 and V2 waves, it is necessary to measure 


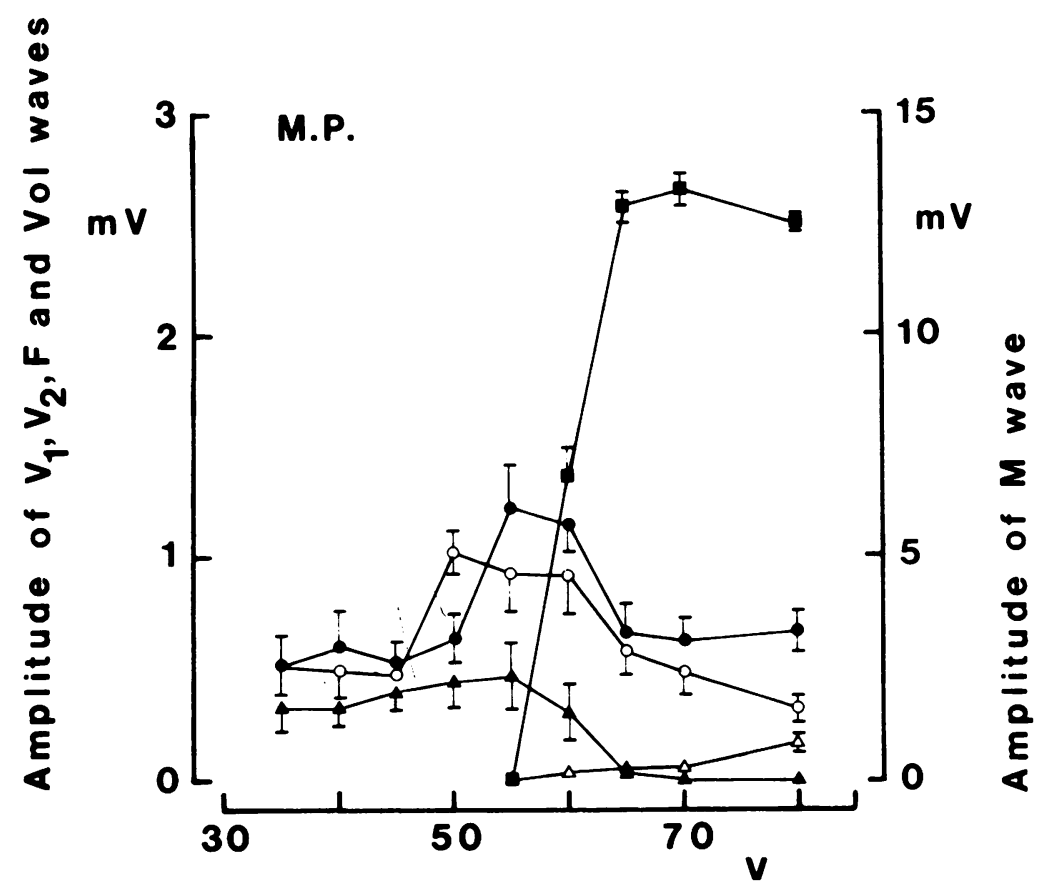

Fig. 2 Relationship between stimulus intensity and the amplitude of evoked responses in one subject. The mean $( \pm S E)$ amplitudes of waves occurring at the average latency of the $V 1(\bullet)$ and $V 2(O)$ waves and $M(\square)$ and $F(\triangle)$ waves are given together with the mean amplitude of MAPs evoked by voluntary activity alone $(\Delta)$ (see text). Note the recruitment of $V 1$ and $V 2$ waves at lower stimulus intensities than the $M$ or $F$ waves. $V=$ stimulus voltage.

the mean amplitude of muscle action potentials occurring as a consequence of the concurrent voluntary activity. It is not sufficient to measure the voluntary activity in the absence of a stimulus because a stimulus of suprathreshold intensity for motor nerves results in an antidromic volley which may well collide with any orthodromic action potentials in the motor nerves. At such stimulus intensities one might expect a reduction in the voluntary activity immediately after the stimulus.

A fixed latency between the $M$ and $V 1$ waves was chosen, and the mean peak-to-peak amplitude of waves, with a negative peak coinciding with this latency, was obtained (termed the Vol wave) from the traces. Waves with a coincident positive peak were not included.

At low stimulus intensities the mean muscle action potential amplitudes at the latencies of the V1 and V2 waves are similar to that of the Vol wave, and all the muscle action potentials are presumably recorded as a consequence of the concurrent voluntary activity. At a stimulus intensity of $50 \mathrm{~V}$ (V2 wave) and $55 \mathrm{~V}$ (V1 wave) the mean amplitude of waves occurring at the latency of the V1 or V2 waves was significantly higher than the mean amplitude of the Vol wave. Clear V1 and V2 waves were obtained at stimulus intensities below motor nerve threshold in all seven subjects. These responses are evoked independently of motor nerve excitation and presumably, therefore, through reflex pathways.

At higher stimulus intensities the motor nerves were excited directly and, as expected, the Vol wave declined in amplitude. The effect of the antidromic volley in the motor nerves on the V1 and V2 waves is unclear (possibly including antidromic collision and antidromic excitation of Renshaw cells) and, therefore, the following experiments were carried out using stimuli of subthreshold intensity for motor nerves only (except where specified).

ESTIMATION OF V1 AND V2 REFLEXES AFFERENT CONDUCTION VELOCITIES

Twenty to 50 electromyogram traces, triggered by a contraction of the thenar muscles to a tension 
of $0.55 \mathrm{~kg}$ were recorded on moving film either after a stimulus of subthreshold intensity for motor nerves or in the absence of a stimulus.

The latencies of the V1 and V2 waves were determined by a trace averaging method. The traces were divided into $2.5 \mathrm{~ms}$ intervals for analysis. The peak-to-peak amplitudes of all MAPs on each trace were measured, and the $2.5 \mathrm{~ms}$ interval into which the negative peaks fell were recorded. The mean amplitude of MAPs in each $2.5 \mathrm{~ms}$ interval was calculated by dividing the total amplitude of all MAPs in each interval by the number of traces. The results for one subject are shown in Fig. 3. Two periods of raised mean wave amplitudes corresponding to the V1 and V2 waves are evident as well as two periods of inhibition.

The responses evoked by subthreshold stimuli to the median nerve at the wrist or the elbow during similar thenar muscle contractions were analysed in the same way. The V1 and V2 waves were evoked at a shorter latency by a stimulus at the elbow in all subjects.

The delay between the stimulus artefact and the midpoint of the modal interval of the $\mathrm{V} 1$ and $\mathrm{V} 2$ response distributions, as shown in Fig. 3, is a rough estimate of mean response negative peak

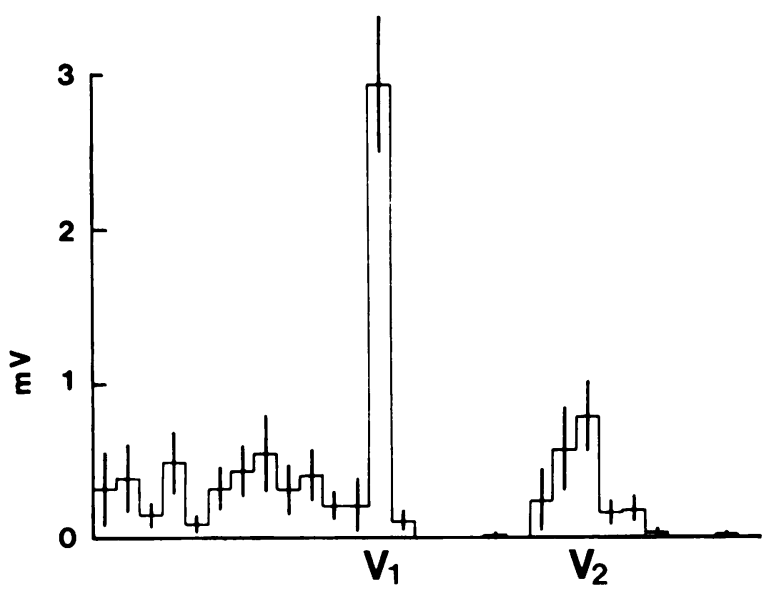

Fig. 3 Mean amplitude $( \pm S E)$ of muscle action potentials in 2.5 ms intervals after a stimulus of subthreshold intensity for motor nerves given to the median nerve at the wrist (upper graph) and in the absence of a stimulus. The abscissa gives the latency from the time at which the stimulus was triggered.

latency. A more accurate estimate may be obtained by considering the intervals on either side of the modal group and calculating a weighted average. The V1 and the V2 wave negative peak latencies obtained in this way with both elbow and wrist stimulation were determined in nine subjects. The latencies of the V1 and V2 waves were used to estimate the V1 and V2 reflexes afferent nerve conduction velocities by dividing the distance between stimulating electrodes at the wrist or elbow sites by the difference in response latency.

The V1 reflex afferent conduction velocity in eight subjects ranged from 52.6 to $84.8 \mathrm{~m} / \mathrm{s}$, with a mean and standard deviation (SD) of 63.72 \pm $9.57 \mathrm{~m} / \mathrm{s}$, while the $\mathrm{V} 2$ reflex afferent conduction velocity was significantly lower $(P<0.001)$ ranging from $30.7-53.9 \mathrm{~m} / \mathrm{s}$ in nine subjects with a mean of $42.5 \pm 8.6 \mathrm{~m} / \mathrm{s}$.

The above method of latency determination avoids subjective discrimination between MAPs evoked through the V1 and V2 reflex pathways and those due to the concurrent voluntary activity. However, a less variable estimate of peak latency may be obtained by finding the mean latency of large amplitude responses. This was possible with the V1 wave as it has a latency varying by less

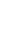

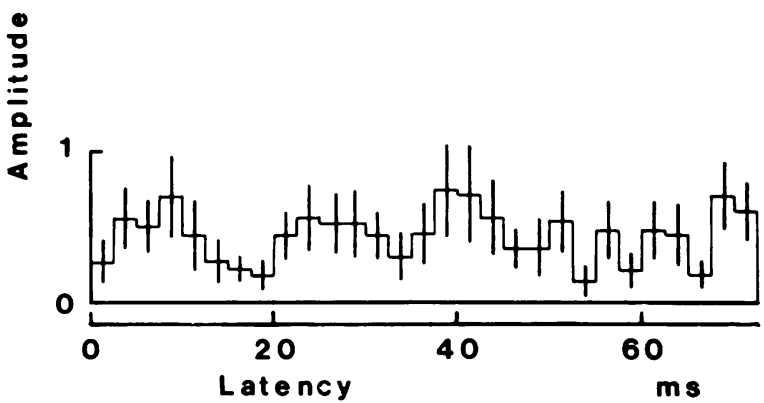


than $5 \mathrm{~ms}$ in any subject, and frequently a large amplitude, and was, therefore, clearly identifiable above the background voluntary activity. However, the difficulty in discriminating the V2 wave from the background noise makes this method unsuitable for determination of the V2 wave latency.

The V1 reflex afferent conduction velocity calculated by this method ranged from $56.0-71.3 \mathrm{~m} / \mathrm{s}$ with a mean of $64.2 \pm(\mathrm{SD}) 4.5 \mathrm{~m} / \mathrm{s}$. The mean conduction velocity is very close to that obtained by the previous method but the range is greatly reduced.

\section{ESTIMATION OF V1 AND V2 REFLEXES CENTRAL DELAYS}

The central delays of the $\mathrm{V} 1$ and $\mathrm{V} 2$ reflexes were estimated by subtracting the calculated peripheral conduction delays (afferent and efferent conduction time and muscle excitation time) from the actual latencies of the V1 and V2 waves.

The afferent nerve conduction velocities for the $\mathrm{V} 1$ and V2 reflexes were determined as above, using the first method for the V2 reflex and the second method for the V1 reflex. The efferent nerve conduction velocity was determined by stimulating at the wrist and the elbow with stimuli of supramaximal intensity for motor nerves and measuring the difference in latency to the peak of the $M$ wave.

The length of the nerve was estimated as the distance from the wrist stimulating electrode to the spine with the arm raised laterally at right angles to the body.

The time taken for the afferent volley to reach the spinal cord was estimated by dividing the length of the nerve by the afferent conduction velocity. Similarly, the time taken for the reflex volley to travel from the spinal cord to the wrist was estimated by dividing the length of the nerve by the efferent conduction velocity. The conduction delays were summed to the terminal delay, the time interval between the stimulus at the wrist and the peak of the $M$ wave, and the calculated total peripheral delay was subtracted from the actual response negative peak latencies to give the central delay.

The V1 reflex central delay had a mean and standard error (SE) of $0.78 \pm 0.84 \mathrm{~ms}$, while the V2 reflex central delay for the same six subjects had a mean of $17.24 \pm(\mathrm{SE}) 1.03 \mathrm{~ms}$.

ORIGINS OF THE V2 AFFERENT FIBRES

The conduction velocity of the V2 afferent fibres suggests that they are sensory fibres of intermediate diameter that could originate in spindle (group II), cutaneous, or joint receptors. It is difficult to eliminate any of these origins but responses of similar latency (allowing for the longer conduction pathway) may be evoked by an electrical stimulus of low intensity to the terminal phallanx of the thumb (Fig. 4). Such responses have been described previously (Caccia et al., 1973). Furthermore, stimuli to the radial nerve at the wrist (distally to the wrist the radial nerve is composed
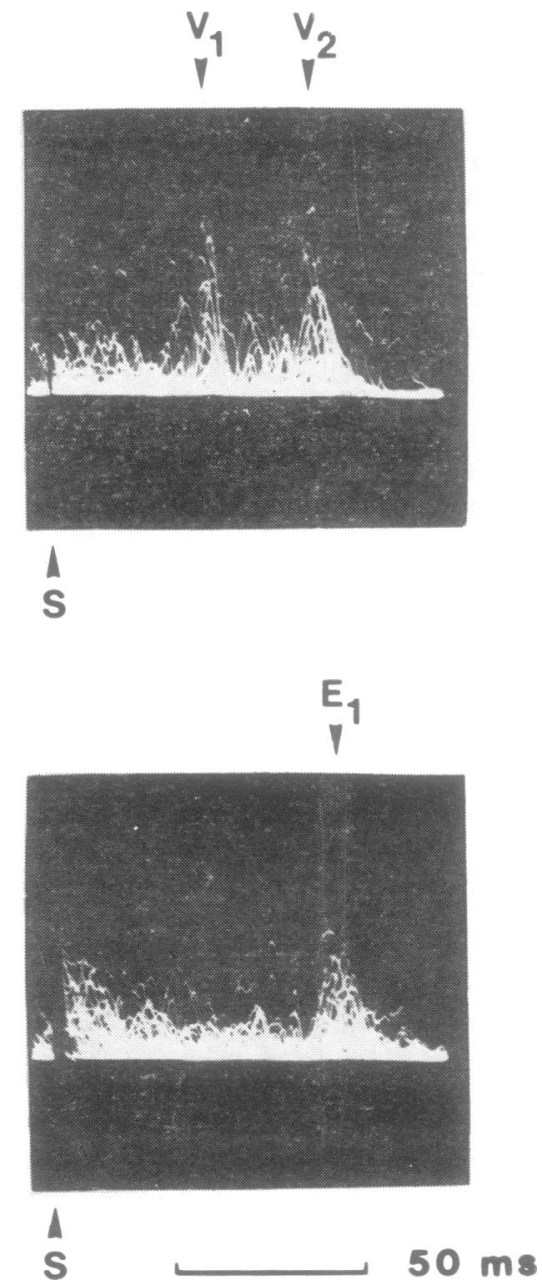

Fig. 4 Superimposed electromyograms recorded from the $A P B$ muscle during voluntary contractions. The electromyograms have been full-wave rectified before display to accentuate the responses. The upper figure shows responses recorded after stimuli of subthreshold intensity for motor nerves to the median nerve at the wrist. The lower figure shows electromyograms recorded after stimuli to the terminal phallanx of the thumb. Note the slightly longer delay of the response, termed the $E 1$ wave, than the $V 2$ wave and the absence in the lower figure of responses corresponding in latency to the $V I$ wave. $S=$ stimulus artefact. 
entirely of cutaneous afferent fibres) evoke responses in the contracting APB muscle of similar latency and variability to the V2 wave (Figs. 1b, 5). These responses were evoked in the absence of $\mathbf{M}$ or V1 waves, even at high stimulus intensities, indicating that nerve fibres of the median nerve were not excited directly by the stimulus.
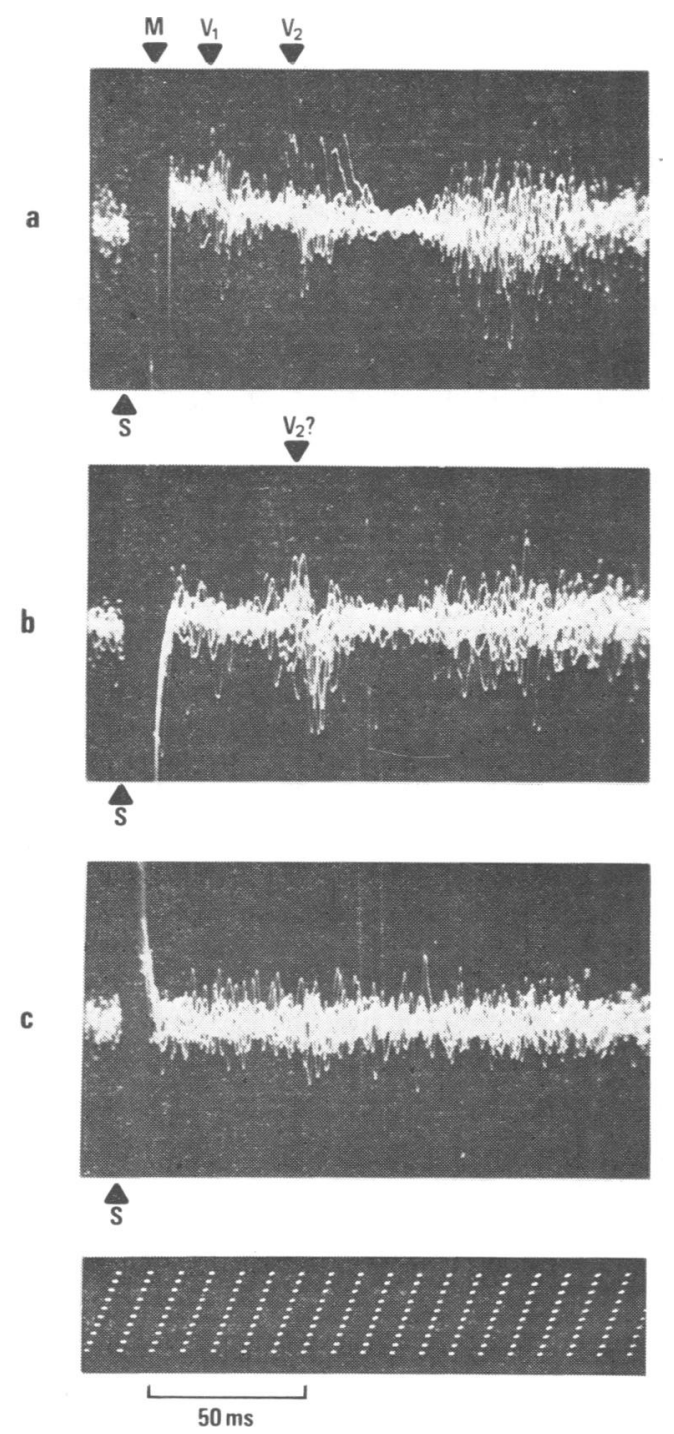

Fig. 5 Fifteen superimposed surface EMG traces recorded from the APB muscle after a stimulus to (a) the median nerve at the wrist $(b)$ the radial nerve at the wrist $(c)$ the skin on the dorsal surface of the wrist, medial to the radial nerve. All stimuli were of the same intensity. Note the response recorded after a stimulus to the radial nerve at the wrist labelled V2?

\section{Discussion}

The V1 and V2 waves were evoked with a shorter latency after a stimulus to the nerve at the elbow than at the wrist indicating a conduction pathway involving the spinal cord. Furthermore, since both responses can be evoked with a stimulus of lower intensity than that required to evoke an $\mathbf{M}$ wave, they originate, unlike the $F$ wave, independently of direct excitation of motor nerves. The V1 and V2 waves are evoked, therefore, through reflex pathways. Upton et al. (1971) did not obtain such a clear separation of $M$ wave and V1 and V2 wave excitabilities. However, these authors used a stimulus pulse duration of 250 or $500 \mu \mathrm{s}$ while in this study a pulse of $1.0 \mathrm{~ms}$ duration was used throughout. It has been shown that longer duration stimuli preferentially excite sensory rather than motor nerves (Erlanger and Blair, 1938; Veale et al., 1973), and, therefore, the stimulus of subthreshold intensity for motor nerves used in this study is likely to have excited a larger number of afferent nerve fibres.

The $\mathrm{V} 1$ reflex has several similarities to the $H$ reflex recorded in the calf muscles. Firstly, it has a short latency. Secondly, the $\mathrm{H}$ reflex afferent conduction velocity has been determined as $60 \mathrm{~m} / \mathrm{s}$ (Magladery and McDougal, 1950), 61 to $70 \mathrm{~m} / \mathrm{s}$ (Mayer, 1963), and 62 to $66 \mathrm{~m} / \mathrm{s}$ (Diamantopolous and Gassel, 1965), values that are very close to the V1 reflex afferent conduction velocity determined here. Furthermore, both the $\mathrm{H}$ and V1 reflex afferent conduction velocities are faster than the efferent nerve conduction velocities determined in the same muscles. Thirdly, the $H$ reflex is only recorded in the muscles innervated by the stimulated nerve (Hoffman, 1918, 1922); the V1 wave is only recorded in the thenar muscles of the hand after a stimulus to the median nerve (Upton et al., 1971). Lastly, the $\mathrm{H}$ reflex may be evoked by a stimulus of subthreshold intensity for motor nerves, and attains a maximal amplitude at intensities below those required to evoke a maximal $\mathbf{M}$ wave (Tábořícová and Sax, 1968). The amplitude of the V1 wave has a similar relationship to the stimulus intensity (Upton et al., 1971; Stanley 1976).

The above evidence is strongly in favour of a similar conduction pathway for the $V 1$ and $H$ reflexes.

The central delays of the $\mathrm{V} 1$ and $\mathrm{V} 2$ reflexes were estimated making two major assumptions: that the efferent and afferent conduction velocities are constant along the lengths of the nerves, and the distance from wrist to spine is a good estimate of peripheral nerve length. However, from the 
above evidence, and from that obtained by Upton et al. (1971), it is reasonable to presume by analogy with the $H$ reflex that the $V 1$ wave is evoked through a monosynaptic reflex pathway. The mean value obtained of $0.7 \mathrm{~ms}$ is within the acceptable range for such a reflex, and suggests that the assumptions made do not affect markedly the estimation of the central delays.

The course of the V2 reflex pathway is more open to conjecture. The latency of the response might suggest a flexion reflex (Shahani, 1969; Cambier et al., 1974) but it has a threshold far below a painful level and a central delay that is far longer. The results presented here suggest the involvement of sensory nerve fibres of smaller diameter than those transmitting the V1 reflex afferent volley. They probably originate in cutaneous or joint receptors though a contribution to the V2 wave due to excitation of spindle afferent fibres cannot be ruled out. This is in contrast to the V2 reflex afferent fibres studied in tibialis anterior muscle of the leg which appear to originate primarily in muscle spindles (Iles, 1977). It is also possible that excitation of cutaneous receptors immediately under the stimulating electrode contributes to the $\mathrm{V} 2$ response.

The long central delay of the V2 reflex suggests a long conduction pathway in the spinal cord. Reflex pathways involving transmission longitudinally within the spinal cord have been described. Shimamura and Livingston (1963) give evidence for a spino-bulbo-spinal reflex pathway, and Marsden et al. $(1972,1973)$ have proposed a spinocerebro-spinal pathway. Whether such pathways are involved in the genesis of the $\mathrm{V} 2$ reflex remains to be determined.

I would like to thank Dr D. J. Aidley, Dr J. C. Brown, and Dr G. Shelton for their many helpful comments, B. Burgoyne for technical assistance, and Ms C. Barlow for secretarial help. This work was supported by a grant from the East Anglian Regional Health Authority Research Fund.

\section{References}

Caccia, M. R., McComas, A. J., Upton, A. R. M., and Blogg, T. (1973). Cutaneous reflexes in small muscles of the hand. Journal of Neurology, Neurosurgery, and Psychiatry, 36, 960-977.

Cambier, J., Dehen, H., and Bathien, N. (1974). Upper limb cutaneous polysynaptic reflexes. Journal of the Neurological Sciences, 22, 39-49.

Diamantopolous, E., and Gassel, M. M. (1965). Electrically induced monosynaptic reflexes in man. Journal of Neurology, Neurosurgery, and Psychiatry, 28, 496-502.
Erlanger, J., and Blair, E. A. (1938). Comparative observations on motor and sensory fibers with special reference to repetitiousness. American Journal of Physiology, 121, 431-453.

Gassel, M. M., and Wiesendanger, M. (1965). Recurrent and reflex discharges in plantar muscles of the cat. Acta Physiologica Scandinavica, 65, 138142.

Hagbarth, K.-E. (1962). Post-tetanic potentiation of myotatic reflexes in man. Journal of Neurology, Neurosurgery, and Psychiatry, 25, 1-10.

Hoffmann, P. (1918). Über die Beziehungen der Sehnenreflexe zur willkürlichen Bewegung und zum Tonus. Zeitschrift für Biologie, 68, 351-370.

Hoffmann, P. (1922). Untersuchungen über die Eigenreflexe (Sehenenreflexe) menschlicher Muskeln. Springer-Verlag: Berlin.

Iles, J. F. (1977). Responses in human pre-tibial muscles to sudden stretch and to nerve stimulation. Experimental Brain Research, 30, 451-470.

McLeod, J. G., and Wray, S. H. (1966). An experimental study of the $\mathrm{F}$ wave in the baboon. Journal of Neurology, Neurosurgery, and Psychiatry, 19, 196-200.

Magladery, J. W., and McDougal, D. B. (1950). Electrophysiological studies of nerve and reflex activity in man. I. Identification of certain reflexes? in the electromyogram and the conduction velocity of peripheral nerve fibers. Bulletin of The Johns Hopkins Hospital, 86, 265-290.

Magladery, J. W., Porter, W. E., Park, A. M., and Teasdall, R. D. (1951). Electrophysiological studies of nerve and reflex activity in normal man. IV. The two neurone reflex and identification of certain action potentials from spinal roots and cord. Bulletin of the Johns Hopkins Hospital, 88, 499-519.

Marsden, C. D., Merton, P. A., and Morton, H. B. (1972). Changes in loop gain with force in the human muscle servo. Journal of Physiology (London), 222, 32-34P.

Marsden, C. D., Merton, P. A., and Morton, H. B. (1973). Is the human stretch reflex cortical rather than spinal? Lancet, 1, 759-761.

Mayer, R. F. (1963). Nerve conduction studies in man. Neurology (Minneapolis), 13, 1021-1030.

Mayer, R. F., and Feldman, R. G. (1967). Observations on the nature of the $\mathrm{F}$ wave in man. Neurology (Minneapolis), 17, 147-156.

Shahani, B. T. (1969). A study in human reflexes. PhD thesis. Oxford University: Oxford.

Shimamura, M., and Livingston, R. B. (1963). Longitudinal conduction systems serving spinal and brainstem co-ordination. Journal of Neurophysiology, 26, 258-272.

Stanley, E. F. (1976). A spects of peripheral nerve and reflex functions in the human hand. PhD thesis. University of East Anglia: Norwich.

Tábořícová H., and Sax, D. S. (1968). Conditioning of $\mathrm{H}$ reflexes by a preceding subthreshold $\mathrm{H}$ reflex stimulus. Brain, 92, 203-212.

Teasdall, R. D., Park, A. M., Languth, H. W., and Magladery, J. W. (1952). Disclosure of normally 
suppressed monosynaptic reflex discharge of spinal motoneurones by lesions of lower brainstem and spinal cord. Bulletin of the Johns Hopkins Hospital, 91, 245-256.

Thomas, J. E., and Lambert, E. H. (1960). Ulnar nerve conduction velocity and $\mathrm{H}$-reflex in infants and children. Journal of Applied Physiology, 15, 1-9.
Upton, A. R. M., McComas, A. J., and Sica, R. E. P. (1971). Potentiation of late responses evoked in muscles during effort. Journal of Neurology, Neurosurgery, and Psychiatry, 34, 699-711.

Veale, J. L., Mark, R. F., and Rees, S. (1973). Differential sensitivity of motor and sensory fibres in human ulnar nerve. Journal of Neurology, Neurosurgery, and Psychiatry, 36, 75-86. 